\title{
Ontogenetic differences in ethanol-induced impairment of context and CS learning
}

\author{
DAVID L. MCKINZIE \\ Indiana University School of Medicine, Indianapolis, Indiana \\ and \\ JIEUN S. LEE, JAMIE H. MCKINZIE, LINDA P. SPEAR, and NORMAN E. SPEAR \\ Binghamton University, Binghamton, New York
}

\begin{abstract}
A hypothesized action of ethanol is that it reduces processing of contextual stimuli. Given previous reports of age-related differences in stimulus selection, in the present study we utilized a conditioned suppression paradigm within an enhanced sensory context to examine the effects of ethanol on context and tone learning in preweanling and adult Sprague-Dawley rats as a function of tonefootshock interval. Although ethanol had some impairing influence on both context and tone conditioning in adults, tone responding nevertheless remained evident, whereas context conditioning was abolished regardless of the tone-footshock interval. In preweanlings, both context and tone responding were abolished by ethanol unless training occurred with contiguous tone-footshock pairings. It is suggested that age-specific modes of encoding are differentially sensitive to the effects of alcohol.
\end{abstract}

Infant and adult animals differ in how they process complex events. When challenged with an unconditioned stimulus (US), adult animals tend to respond selectively to stimuli most predictive of the US. A common consequence of such stimulus selection is overshadowing: attenuated responding to a single element when it occurs in a conditioned stimulus (CS) compound. Infant animals differ from adults in that they do not respond to stimuli selectively. In direct ontogenetic comparisons between infant and adult rats, with different stimulus compounds of varying modalities, adults typically exhibit overshadowing, whereas infants show less overshadowing and often demonstrate potentiated responding (Hinderliter \& Misanin, 1988; Kucharski \& Spear, 1985; Mellon, Kraemer, \& Spear, 1991; Spear \& Kucharski, 1984a).

The disposition of infants to express potentiation when they are tested on a single element of a compound stimulus does not appear to be specific to discrete stimuli. Potentiation of context conditioning by CS learning has also been observed. McKinzie and Spear (1995) have examined the effects of CS-US interval and context salience on context and CS learning in preweanling and adult rats. In preweanlings, both context and CS conditioning were strongest when forward CS-US pairings were administered in an enhanced sensory context; this indicated reciprocal potentiation. Adult rats, on the other hand, responded selectively to either the context or the CS, depending on which was most predictive of the US.

This research was supported by NIAAA Grants 5RO1 AA06634 and 5RO1AA 10223 to N.E.S. and by NIAAA Grant 1 F31 AA05349 to D.L.M. Correspondence should be addressed to D. L. McKinzie, Department of Psychiatry, Indiana School of Medicine, Indianapolis, IN 46202 (e-mail: dmckinzi@indyunix.iupui.edu).
These interactions between context conditioning and CS conditioning may be understood in terms of how a preweanling (infant) encodes the joint occurrence of a target CS within that context. It has been suggested that infants may encode stimuli primarily on the basis of amodal stimulus attributes (Mellon et al., 1991; Spear \& McKinzie, 1994; Spear \& Molina, 1987; Turkewitz, 1994; Turkewitz \& Mellon, 1989). By ignoring the energy source (i.e., modality) of sensory stimuli and attending to invariant properties common to all modalities (e.g., intensity), the infant could greatly reduce processing load. When presented with a complex multisensory event, an infant may respond to such amodal stimulus characteristics as the combined net intensity of stimuli at a given moment, or to other amodal attributes such as familiarity or affect. Adults, on the other hand, appear more likely to differentiate between stimuli from varying energy sources.

One strategy for examining ontogenetic differences in stimulus selection processes is to use pharmacological manipulations. For example, ethanol has been found to alter attentional processes, reduce behavioral variability, and increase response perseveration (Devenport, 1984; Devenport, Merriman, \& Devenport, 1983; Devenport, Stidham, \& Hale, 1989; Erblich \& Earleywine, 1995; Jalava, Mattila, Tarssanen, \& Vanakoski, 1995; Maier \& Pohorecky, 1986; Marriott, Alpern, \& Crabbe, 1974; Sahgal, Eckberg, Howell, \& Iverson, 1980). These effects of ethanol may be attributable to a reduction in the processing of incidental stimuli. Devenport and Cater (1986) administered either saline or ethanol to adult rats prior to training in a radial maze task. After successful acquisition had been determined in both groups, a context shift was implemented. Although saline-treated rats expressed a context shift decrement when tested in the novel con- 
text, rats trained under the influence of ethanol did not, which suggests that ethanol acted to limit processing of incidental contextual stimuli (Devenport \& Cater, 1986).

If ethanol limits or reduces processing of context, ontogenetic differences in context and CS learning may be altered. A recent study examined the influence of ethanol on the learning of context and CS in preweanling rats (McKinzie, Lee, Bronfen, Spear, \& Spear, 1994). In a state-dependent design, preweanlings were trained with paired or unpaired CS-US presentations after intragastric (i.g.) administration of $1.2-, 1.6-$, or $2.0-\mathrm{g} / \mathrm{kg}$ ethanol $(17 \% \mathrm{v} / \mathrm{v})$ or an equivalent volume of water. Although a low dose of ethanol $(1.2 \mathrm{~g} / \mathrm{kg})$ had no effect on context or CS conditioning, the intermediate dose $(1.6 \mathrm{~g} / \mathrm{kg})$ disrupted conditioning to the context. With the highest dose $(2.0 \mathrm{~g} / \mathrm{kg})$, both context and CS learning were disrupted, depending on when ethanol was administered (training, testing, or both). This indicates that, at least in preweanlings, ethanol can affect more than just the learning of the context.

The purpose of the present study was to extend the original findings of McKinzie et al. (1994). We examined context and CS learning in both preweanlings and adults. Although the influence of ethanol on context and CS conditioning has been examined separately for both infants and adults, this is the first study in which the effects of ethanol on context and CS learning have been directly compared in rats of different ages. We used an enhanced sensory context to accentuate age-related differences in stimulus selection. Our hypothesis was that ethanol would preferentially disrupt context conditioning in adults without affecting conditioning to the CS. The rationale was that if ethanol serves to reduce processing of incidental stimuli, then learning for only relatively discrete and salient cues (i.e., tone CS) would be maintained. We were also interested in whether a salient context would attenuate the deleterious effects of ethanol on context learning in preweanling rats. We hypothesized that an especially salient context would dispose the infant to attend to amodal stimulus properties of the environment because the infant is overwhelmed by the intense multisensory stimulation under these conditions and is incapable of stimlus processing on a modal basis. A shift to amodal encoding reduces stimulus processing load because the infant responds to just a few stimulus attributes that are invariant across modalities. Within this framework, an enhanced context may attenuate the impairing influence of ethanol on context and CS conditioning, because amodal processing requires less attentional resources than does modal encoding and, therefore, may be less susceptible to the attention-impairing effects of ethanol.

In Experiment 1, we examined the effects of an enhanced sensory context and ethanol pretreatment on context and CS learning in preweanling rats as a function of CS-US interval. Experiment 2 established doses yielding comparable blood alcohol concentrations for preweanlings and adults during conditioning. In Experiment 3, the effects of ethanol on context and CS conditioning were assessed in adults given CS-US conditioning in an enhanced context. Since adults tend to respond selectively to stimuli and ethanol has previously been shown to impair the consequences of a change in context (Devenport \& Cater, 1986), ethanol administration to adults was expected to be more detrimental to context conditioning than to CS conditioning.

\section{EXPERIMENT 1}

Context and CS conditioning were examined in preweanling rats after a $1.6-\mathrm{g} / \mathrm{kg}$ administration of $17 \% \mathrm{v} / \mathrm{v}$ ethanol or an equivalent volume of only the vehicle (water). Conditioning with pairings of CS and US separated by trace intervals of 0 or $10 \mathrm{sec}$ was evaluated against control conditions for the CS-US pairing (unpaired) and for the context-US pairing (context control). Training and testing were conducted within an enhanced sensory context because of previous findings of strong age-related differences in stimulus selection under these conditions (McKinzie \& Spear, 1995). The trace interval group also served as a comparison so that we could assess the effects of ethanol when CS and US presentations were temporally discontiguous. We hypothesized that context and CS learning would be least affected by ethanol in pups trained with 0 -sec CS-US pairings, because at this interval, the likelihood of amodal processing should be greatest (i.e., at the time of maximum sensory stimulation; context + CS + US).

\section{Method}

Subjects. The subjects were 91 male and female 17-day-old Sprague-Dawley-derived rat pups. Preweanlings were housed with both parents and littermates in standard opaque maternity cages that were partially filled with pine shavings. All rats were housed in a temperature-controlled vivarium maintained on a $16: 8$-h light:dark cycle with light onset occurring at $0600 \mathrm{~h}$. Food and water were available ad lib. Training and testing were conducted during the light portion of the light:dark cycle.

Apparatus. During training, rats were exposed to two different contexts. Context 1 , termed the "control" context, was located in a room different from that for the training and testing contexts. A white $54 \times 40 \times 46 \mathrm{~cm}$ sound-insulated box (Coulbourn) contained a $38 \times 30 \times 7 \mathrm{~cm}$ shock grid, which rested on the compartment floor. The grid bars were $3 \mathrm{~mm}$ in diameter, spaced $3.5 \mathrm{~mm}$ apart. A clear Plexiglas chamber $(28 \times 20.5 \times 25.5 \mathrm{~cm})$, partitioned into three equal-sized compartments, was placed on the grid floor. A Grason-Stadler shock generator delivered a 1-mA, 1-sec scrambled footshock to appropriate groups. The subjects were placed in the two outer compartments, and visual contact was prevented by pink construction paper attached to the walls of the middle compartment. The chamber door remained open, and subjects were exposed to ambient room lighting. Orange odorant ( $1.0 \mathrm{cc}$; Spectrum-California) was placed under the shock grid, and a fine wire mesh top was placed over the top of the chamber.

Context 2 was the context in which CS training and testing occurred. Rat pups received training in an enhanced sensory context, which consisted of a sound-insulated chamber (Coulbourn; $54 \times$ $40 \times 46 \mathrm{~cm}$ ) that contained the training and test apparatus. A 38 $\times 30.5 \mathrm{~cm}$ shock grid with grid bars $3 \mathrm{~mm}$ in diameter spaced $1 \mathrm{~cm}$ apart was situated on the compartment floor. A $28 \times 20.5 \times$ 
$25.5 \mathrm{~cm}$ black Plexiglas chamber, divided into two equal compartments, was placed on the grid floor. The CS was a 15-sec tone of $2000 \mathrm{~Hz}$ that measured $80 \mathrm{~dB}$ (A-scale) from the grid floor. The tone was delivered from three speakers situated $34 \mathrm{~cm}$ above the grid floor. A Grason-Stadler shock generator delivered a 1-mA, 1sec scrambled footshock to appropriate groups. A ventilation fan of $72 \mathrm{~dB}$ provided background noise, and a small houselight of $1.5 \mathrm{~W}(120 \mathrm{~V})$ provided dim ambient light in the apparatus. An ambient lemon odor ( $2 \mathrm{cc}$; Humco) was placed under the grid floor, and three flashing lights $(7.5 \mathrm{~W}, 120 \mathrm{~V})$ remained on during the experiment. The light bulbs were $29 \mathrm{~cm}$ above the grid floor and flashed on and off $(0.5 / 0.5 \mathrm{sec})$.

Testing was conducted in the training context $24 \mathrm{~h}$ later. The test chamber had physical dimensions identical to those of one of the partitioned conditioning compartments, except that two circular disks, each of which was $2.5 \mathrm{~cm}$ in diameter, were added on one wall; these were centered $1.5 \mathrm{~cm}$ from the grid floor and spaced $4.0 \mathrm{~cm}$ apart. The disks were the transmitter and receiver for an ultrasonic activity-monitoring device, described by Moye and Rudy (1985). The device emits an ultrasonic signal, which is detected by a receiver. Frequency discrepancies are positively correlated with the activity of the subject; each discrepancy causes a closure of an electromechanical relay, resulting in one activity count. The sensitivity of the device is calibrated so that movements requisite for respiration are not generally detected. While subtle movements of the head and vibrissae are detected, more activity counts are recorded during whole body movements such as locomotion and rearing.

Procedure. Preweanlings were removed from the home cage, weighed, and placed in a holding cage with one other littermate. The pups were then intubated with a $1.6-\mathrm{g} / \mathrm{kg}$ dose of $17 \% \mathrm{v} / \mathrm{v}$ ethanol solution or an equivalent volume of water. Immediately following intubation, the rats were placed in the exposure control context. Context control groups received 16 unsignaled footshocks presented on a 100 -sec variable-time (VT) intertrial interval (ITI) schedule. All other groups received context-only exposure for $30 \mathrm{~min}$. Following $30 \mathrm{~min}$ in the control context, the rats were taken immediately to the training context. Paired subjects received 16 tone-footshock trials (with either a 0 - or a 10 -sec CSUS interval) presented on a $100-$ sec VT ITI schedule. Unpaired animals were given quasi-random unpaired presentations of the tone and footshock, with the constraint that at least $60 \mathrm{sec}$ must separate each tone and footshock. Context controls received CSonly trials, presented on the same temporal schedule as that for the paired subjects.

Preweanlings were tested individually for context and CS conditioning $24 \mathrm{~h}$ later in the training context. The pups were intubated with the same solution given prior to training and allowed to wait in a holding cage for $30 \mathrm{~min}$. After a $1-\mathrm{min}$ adaptation period following placement into the test chamber, context conditioning was assessed by recording the latency to accumulate 100 activity counts. Following the measure of context conditioning, rats remained in the presence of the context alone for an additional 100 counts. This interval was added to further extinguish any residual conditioning to the context. A baseline period then followed, which was designed to establish a consistent rate of activity before CS onset. During the baseline period, latency to reach three consecutive 25 count blocks was recorded. If the latency to reach the fourth 25 count block was within two standard deviations of that for the previous three blocks, a stable baseline was determined and the CS was presented. If the fourth block was not within two standard deviations of the previous three blocks, the baseline period was extended for another 25-count block. This was continued until the criterion of two standard deviations was met.

Statistical analysis. Experiments 1 and 3 were designed specifically to address the effects of ethanol on context and CS learning. We have reported previously the effects of an enhanced train- ing context on CS and context learning in preweanling and adult rats (McKinzie \& Spear, 1995). Since the focus of Experiments 1 and 3 was to determine the effects of acute ethanol administration on context and CS learning and could be addressed by comparing a few critical groups, pairwise comparisons were used to analyze the data (Hays, 1988; Keppel, 1982; Myers \& Well, 1991). Comparisons were limited to experimental groups versus appropriate control groups and between experimental groups differing only in drug administration (e.g., 0 trace water vs. 0 trace ethanol). The error term value from an omnibus group $\times$ drug analysis of variance (ANOVA) was used for each planned comparison. If the number of planned comparisons exceeded the natural limit ( $d f$ of treatment groups), a modified Bonferroni test was used to control for increased Type I error (Keppel, 1982).

\section{Results}

Context conditioning. The time to reach the first 100 activity counts after a 1 -min adaptation period was used as the context conditioning measure. This value was then converted to a $\log$ (base 10 ) score to normalize the sample distribution. The following comparisons were planned: (1) paired (0-sec CS-US interval), trace (10-sec CS-US interval), and unpaired versus context control groups receiving the same drug administration, and (2) vehicle versus ethanol groups trained with the same CS-US interval. Planned comparisons were assessed for significance $(\alpha=.05)$ against a modified Bonferroni correction value.

The top panel of Figure 1 shows context conditioning in vehicle- and ethanol-treated preweanling rats. In comparison with the context controls, paired and trace groups administered vehicle exhibited conditioning to context $[F(1,83)=9.25$, and $F(1,83)=10.21$, respectively], whereas vehicle-treated unpaired pups did not $[F(1,83)=$ 3.97]. In groups receiving ethanol administrations prior to training and testing, only the paired animals appeared to be unaffected by ethanol $[F(1,83)=9.36]$. When they were compared with the context controls, neither the trace nor the unpaired ethanol-treated groups exhibited any context conditioning. Differences between ethanoland saline-treated trace and unpaired groups approached but did not reach statistical significance. The observed differences in conditioning did not appear to be an artifact of ethanol-induced suppression of activity, because the context controls exhibited similar activity levels regardless of drug treatment.

Tone conditioning. Since baseline activity prior to CS onset was found to be comparable in all groups, as evidenced by an omnibus group $\times$ drug ANOVA (all $p$ values $>.44$ ), the data were expressed as the latency to emit the first 100 activity counts in the presence of the tone CS. As seen in the bottom panel of Figure 1, the paired and trace vehicle-treated rats expressed robust tone conditioning $[F(1,83)=15.84$, and $F(1,83)=7.48$, respectively]. The preweanlings treated with ethanol, however, exhibited tone conditioning only when trained with a 0 -sec CS-US interval (Group Paired) $[F(1,83)=15.70]$ Ethanol impaired conditioning in the trace group, as was evidenced by no difference from the context controls and significantly lower latencies (less fear) than those of vehicle-treated trace animals $[F(1,83)=6.97]$. 


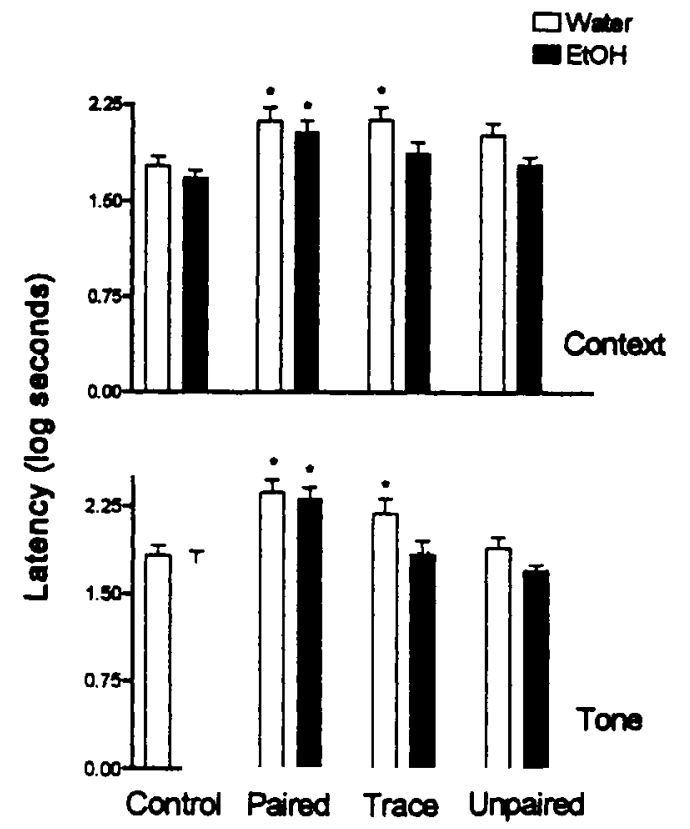

Figure 1. Subjects were 17-day-old rat pups given a $1.6-\mathrm{g} / \mathrm{kg}$ dose of either $17 \% \mathrm{v} / \mathrm{v}$ ethanol solution or equivalent volume of water. Higher latency values indicate greater conditioning levels. Top: Mean time (log sec) to reach the first 100 activity counts following a 1 -min adaptation period (context conditioning) as a function of CS-US interval and drug state. Bottom: Mean time (log sec) to reach the first 100 activity counts following the onset of the CS (tone conditioning). *Significantly different from control group. Vertical lines represent standard errors.

These results suggest that ethanol had a differential influence on context and CS conditioning, depending on CS-US interval. As we have indicated previously (McKinzie \& Spear, 1995), training and testing in an enhanced sensory context produced strong context and CS conditioning in preweanlings whether or not a trace interval separated the CS and US. When ethanol was administered, however, both context and CS conditioning were disrupted if a trace interval was imposed between CS and US presentations (Group Trace). Ethanol had a similar attenuating effect on context conditioning in the trace group. Experiments 2 and 3 were conducted to determine whether similar results would be observed in adult rats.

\section{EXPERIMENT 2}

In order to compare the effects of ethanol on preweanlings and adults, comparable blood alcohol levels are desirable. For instance, if adults should show less of a drug effect on behavior than that found in infants given the same dose of ethanol, the effect could be due to adults' being less intoxicated than infants. Several studies have shown ontogenetic differences in the metabolism of alcohol (Hollstedt \& Rydberg, 1985; Kelly, Bonthius, \& West, 1987; Rajachandran, Spear, \& Spear, 1993). The present study was conducted to establish comparable blood alcohol levels in preweanlings and adults at the time of training.

\section{Method}

Subjects. The subjects were naive 17-day-old and 60- to 90day-old rats ( $n=8$ /age group) born and raised in our Binghamton vivarium. The preweanlings were raised identically to those described in Experiment 1 . The adult rats were weaned at 21 days of age and placed in standard triple-sized hanging wire cages with same-sex conspecifics ( $n=4-6 /$ cage). Food and water were available at all times during rearing.

Procedure. The rats were assigned to one of two groups that differed in time following intubation $(30$ or $60 \mathrm{~min}$ ). Thirty and $60 \mathrm{~min}$ were used since they approximated the time points designating the beginning and end of the training session in Experiment 1 . After group assignment, the rats were intubated with either a 1.6 - (infants) or a 2.0 - (adults) $\mathrm{g} / \mathrm{kg}$ dose of $17 \% \mathrm{v} / \mathrm{v}$ ethanol. The $2.0-\mathrm{g} / \mathrm{kg}$ dose for adults was estimated as probably equivalent in effect to the $1.6-\mathrm{g} / \mathrm{kg}$ dose for preweanlings, on the basis of unpublished preliminary data. At the appropriate time point following intubation, subjects were decapitated and the trunk blood was transferred into a heparinized beaker. During vortexing, $200 \mu \mathrm{l}$ was pipetted into a centrifuge tube containing $1.8 \mathrm{ml}$ of trichloroacetic acid. Each sample remained on ice until all the samples were collected. The samples were then centrifuged at $2,000 \mathrm{rpm}$ for $5 \mathrm{~min}$ in order to obtain a clear supernatant. From the supernatant, $10 \mu \mathrm{l}$ were added to two cuvets, one of which contained $100 \mu \mathrm{l}$ of alcohol reagent solution (test sample; Sigma Chemical Co. Procedure No. 333-UV), and the other, $100 \mu l$ of double distilled water (blank sample). The solutions were allowed to incubate for $10 \mathrm{~min}$ and were assessed for NADH formation using a spectrophotometer set at $340 \mathrm{~nm}$. To determine alcohol concentrations, each blank was subtracted from its own test sample and $\mathrm{mg} / \mathrm{dl}$ blood alcohol levels were calculated.

\section{Results}

An age ( $\mathrm{P} 17$ or $\mathrm{P} 60-90) \times$ time ( 30 or $60 \mathrm{~min}$ ) betweengroups ANOVA was conducted on the $\mathrm{mg} / \mathrm{dl}$ blood alcohol levels. Figure 2 illustrates $\mathrm{mg} / \mathrm{dl}$ blood alcohol levels for both preweanlings and adults. There were no significant differences between the alcohol levels in preweanlings and adults, and there was no interaction between age and time period; only a trend for time period approached significance $(p>.07)$. These results indicate that blood levels of alcohol attained in the circumstances for preweanlings given $1.6 \mathrm{~g} / \mathrm{kg}$ and adults given $2.0 \mathrm{~g} / \mathrm{kg}$ are comparable.

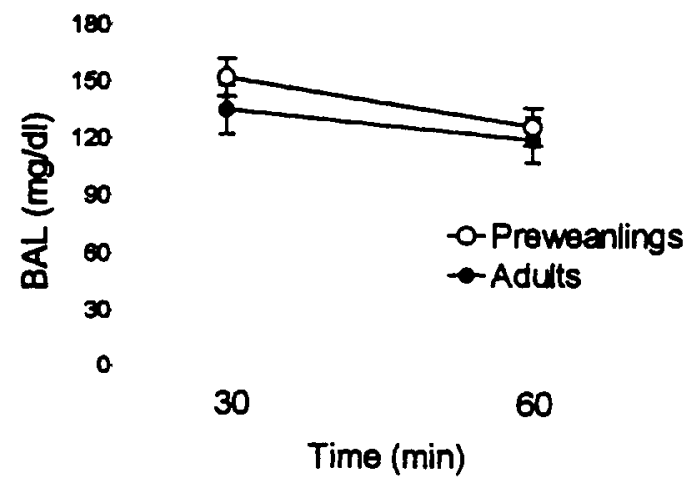

Figure 2. Blood alcohol levels $(\mathrm{mg} / \mathrm{dl})$ for preweanlings and adults at 30 and 60 min following ethanol administration. Subjects were given a $17 \% \mathrm{v} / \mathrm{v}$ ethanol solution $(1.6 \mathrm{~g} / \mathrm{kg}$ for preweanlings and $2.0 \mathrm{~g} / \mathrm{kg}$ for adults). Vertical lines represent standard errors. 
Although no statistically significant differences in blood alcohol levels existed between infants and adults, it is apparent that the adults' levels were somewhat below those of the infants. A higher dose of ethanol was not used in adults, out of concern for the adverse depressive consequences of ethanol intoxication. Since conditioned suppression of activity was the dependent measure in these studies, it was of methodological importance not to have ethanol-related motor coordination disturbances in adults, which could have contributed to interpretative difficulties. This concern was less consequential for preweanlings, since they seem more resistant to the effects of ethanol on motor coordination (Hollstedt \& Rydberg, 1985; Hollstedt, Olsson, \& Rydberg, 1980).

\section{EXPERIMENT 3}

The purpose of this experiment was to examine the influence of ethanol on context and CS conditioning in the adult rat. Adults were trained and tested in an enhanced context as were preweanlings in Experiment 1 . The only procedural deviation from Experiment 1 was the use of a $60-\mathrm{sec}$, instead of a $10-\mathrm{sec}$, trace interval. A longer trace interval for adults was used in an effort to equate CS conditioning across ages, in view of age-related differences in trace conditioning (McKinzie \& Spear, 1995; Moye \& Rudy, 1987). This particular trace interval was determined in preliminary experiments and was selected because context and CS conditioning with this CS-US interval have been found to be comparable to that in preweanlings with a $10-\mathrm{sec}$ trace interval. This condition allowed assessment of whether ethanol differentially affected context and CS conditioning.

\section{Method}

Subjects and Apparatus. The subjects were 79 male and female rats ranging from 60 to 90 days of age. The rats were of the same colony stock and had the same rearing conditions as in Experiment 2 . The same apparatuses and context configurations were used as in Experiment 1, except that a larger grid floor was used for the adults. The grid bars were $5 \mathrm{~mm}$ in diameter, spaced $10 \mathrm{~mm}$ apart.

Procedure. The training and testing procedures were identical to those of Experiment 1, except that the adults were given 2.0$\mathrm{g} / \mathrm{kg}$ administrations of ethanol $(17 \% \mathrm{v} / \mathrm{v})$ or an equivalent volume of water. The rats were intubated and placed in the control context for $30 \mathrm{~min}$. The unpaired, paired, and trace groups received exposure only to this context, whereas the context controls received 16 US-only presentations. Upon completion of the $30 \mathrm{~min}$, the rats were taken immediately to the training context. The paired, trace, and unpaired rats received CS and US presentations, and the context controls were administered CS-only trials. Testing was conducted $24 \mathrm{~h}$ later in the training context. The testing procedures were identical to those in Experiment 1.

\section{Results}

Context conditioning. Consistent with previous evidence, the top panel of Figure 3 shows that context conditioning in vehicle-treated animals became stronger the more the CS-US presentations were separated. Although context learning was not significant in the paired group, significant fear of the context was acquired in the trace and unpaired groups $[F(1,71)=12.35$, and $F(1,71)=$ 15.96 , respectively]. When ethanol was administered, however, context learning was not expressed in any of the groups. Regardless of the CS-US interval, the latencies of the experimental animals given ethanol did not differ from those of the context controls. Additionally, both the trace and the unpaired rats administered ethanol showed significantly less conditioning than did their respective vehicle-treated groups $[F(1,71)=12.20$, and $F(1,71)=$ 17.36 , respectively].

Tone conditioning. Baseline activity prior to tone onset did not differ between treatment groups (all $p$ values $>$ .25). As can be seen in the bottom panel of Figure 3, strong tone conditioning was evident in the paired and trace groups given vehicle administrations $[F(1,71)=48.32$, and $F(1,71)=28.34$, respectively]. The effects of ethanol in the paired animals was only mildly disruptive, because tone conditioning was maintained $[F(1,71)=25.64]$ and no differences were observed between the ethanol-treated and vehicle-treated rats. However, ethanol had an impairing influence on tone conditioning in the trace group, as is evidenced by a failure to differ from the control group and by a significant reduction in conditioning when the trace group was compared with their vehicle-treated counterparts $[F(1,71)=7.29]$.

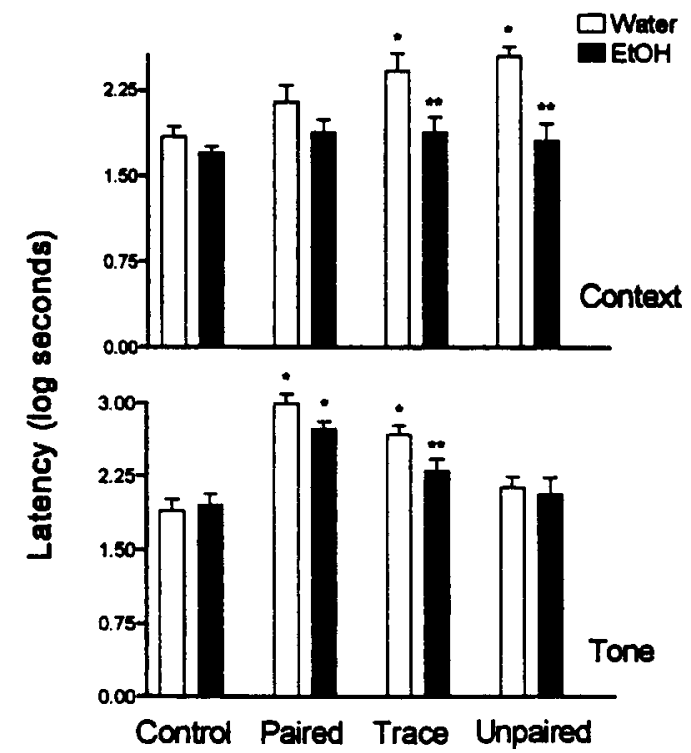

Figure 3. Subjects were adult rats given a $1.6-\mathrm{g} / \mathrm{kg}$ dose of either $17 \% \mathrm{v} / \mathrm{v}$ ethanol solution or equivalent volume of water. Higher latency values indicate greater conditioning levels. Top: Mean time (log sec) to reach the first 100 activity counts following a 1-min adaptation period (context conditioning) as a function of CS-US interval and drug state. Bottom: Mean time (log sec) to reach the first 100 activity counts following the onset of the CS (tone conditioning). *Significantly different from control group. ${ }^{*}$ *Significantly different from groups given same CS-US interval under alcohol intoxication. Vertical lines represent standard errors. 


\section{GENERAL DISCUSSION}

In adults, vehicle-treated animals responded similarly to adult subjects in previous reports (Marlin, 1981; McKinzie \& Spear, 1995). Selective context conditioning was found in unpaired animals, whereas paired animals exhibited only CS learning. Moderate context and CS conditioning were observed in the vehicle-treated trace group. The results of the ethanol data suggest that ethanol disrupts the processing of contextual stimuli preferentially, but not selectively. Although CS conditioning was impaired by ethanol when a trace interval was imposed, the effects of ethanol on context learning were especially strong, in that conditioning was disrupted even under conditions that typically promote strong context responding (i.e., unpaired CS and US presentations). Greater ethanolinduced impairment of context conditioning is consistent with the view that ethanol reduces the processing of incidental stimuli (Devenport \& Cater, 1986). For example, the often found reduction in behavioral flexibility and enhanced response perseveration following ethanol administration may be due to a failure to process extraneous stimuli contained within the environment.

The pattern of ethanol effects was somewhat different for preweanling rats. The facilitative effects of an enhanced sensory context were replicated, in that vehicle-treated paired animals exhibited both context and CS conditioning. Ethanol administrations, however, impaired both context and CS conditioning unless the pups received contiguous CS-US pairings. It is important to note that the absence of an ethanol effect occurs only in an enhanced sensory context. A previous study with similar procedural parameters, but within a relatively plain context, found that a $1.6-\mathrm{g} / \mathrm{kg}$ dose of ethanol selectively disrupted context conditioning in paired rat pups (McKinzie et al., 1994). It may seem paradoxical that a context with a richer source of sensory stimulation should attenuate the deleterious effects of ethanol. We propose, however, that the enhanced context promoted amodal processing in preweanlings. When infants were presented with contiguous CS-US pairings in a sensory-rich context, they were unable to respond selectively and reverted to amodal processing. Through responding predominantly to the combined intensity of context and CS at the time of US onset and ignoring other attributes of the CS, demands on stimulus processing would be reduced, and this processing would be more resistant to the detrimental effects of ethanol.

The view that infants are more likely than adults to process sensory information in an amodal manner is not new and has been discussed elsewhere (Karmel, Gardner, \& Magano, 1991; Mellon et al., 1991; Spear \& Molina, 1987; Turkewitz, 1994). Turkewitz, Lewkowicz, and colleagues have conducted extensive research to support the hypothesis that infant animals and humans often ignore the modality in which a stimulus is presented and respond instead to the net intensity of sensory stimulation (Lewkowicz, 1994; Turkewitz, Lewkowicz, \& Gardner,
1983). Although most of the data in support of this hypothesis have been obtained with the use of discrete visual and auditory stimuli or conditioning to odors or flavors, we propose that contextual stimuli also are susceptible to amodal encoding by infants. The net intensity of a nominal CS may summate with that of context to become a single, more intense stimulus. Responding occurs to the intensity-based configuration rather than to the individual elements that it comprises.

The proposal that amodal processing is more likely under conditions of information overload, provided in the present study by enhanced context, is suggested by previous research. We have consistently found in our laboratory that under conditions requiring rapid or complex cognitive processing (e.g., few training trials or simultaneous CS compounds), the infant rat seems more likely to demonstrate evidence for amodal processing. If processing demands are reduced by giving more training or separating CS compounds into temporally distinct elements, infants respond in an adult-like manner (Spear \& Kucharski, 1984a, 1984b; Spear \& McKinzie, 1994). Perhaps infants attempt initially to process modally but revert to amodal stimulus selection when processing capabilities are exceeded. For example, if a tone presented in a relatively familiar context is followed by a light, the infant may be able to differentiate between the energy sources and perceive a tone followed by a light. If, however, the stimuli are presented simultaneously or the tone occurs in a sensory-rich context, the infant may not have the cognitive resources to distinguish between stimuli on the basis of modalities. In this case, responding to stimulus attributes common to all modalities is an adaptive alternative. It should also be noted that although we have discussed mainly the amodal property of intensity, other amodal stimulus characteristics exist and might be utilized (e.g., duration, form, familiarity, rhythm; Spear \& Molina, 1987).

Neural mechanisms underlying infantile processing such as perceptual configuration and amodal encoding, which have seemed to explain effects such as potentiation in preweanlings, have not been studied. One neuroanatomical area likely to be involved in the present effects is the hippocampus. Electrophysiological data indicate that relatively low concentrations of ethanol inhibits NMDA-stimulated calcium channels in mature (Daniel, 1992; Lovinger, White, \& Weight, 1989, 1990) and immature hippocampal neurons (Swartzwelder, Wilson, \& Tayyeb, 1995). Ethanol also affects behaviors mediated by hippocampal function (Devenport \& Merriman, 1983; Gibson, 1985; Maier \& Pohorecky, 1986; Mathews, Simson, \& Best, 1996; but see Devenport \& Hale, 1989). An immature hippocampus has been also implicated in ontogenetic differences in stimulus processing (Carew \& Rudy, 1991; Rudy, 1991; Rudy \& Morledge, 1994). Moreover, hippocampal lesions differentially affect conditioning to context and discrete auditory cues (Kim \& Fanselow, 1992; Phillips \& LeDoux, 1992 ) and often promote infantile-like processing in le- 
sioned adults (Rudy \& Sutherland, 1989; Spear, McKinzie, \& Arnold, 1994).

Rudy and colleagues have proposed that ontogenetic differences in learning can be attributed to a late-maturing hippocampal system (Rudy, 1991, 1992). Specifically, hippocampally immature infants or hippocampally lesioned adults are viewed as capable of forming only simple associations between elements in their environment, whereas normal adults are viewed as capable of forming relational representations of stimuli. Rudy consistently finds that infants are incapable of solving relational tasks, such as negative/transverse patterning and conditional discriminations, that require configural associations of two or more elements (Alvarado \& Rudy, 1992; Rudy, Keith, \& Georgen, 1993). As a result, infants can learn simple context-US associations but have been found unable to "disambiguate" a learning episode (Carew \& Rudy, 1991).

We suggest that under most situations, stimulus selection based on sensory modality is needed for configural learning. Complex stimuli are often composed of multisensory information and require differentiation of the various sensory inputs in order for conjunctive associations to be formed between elements. In our paradigm, in which preweanlings express strong context and CS learning as a result of being trained in a salient context, we suggest that infants process information at an elemental level but are attending to amodal stimulus properties. Instead of associating separable lights, tones, and odors with an aversive footshock, infants may associate a "bright-loud-odor" with the footshock. Differential effects may be found in infants capable of only elemental processing, depending on whether elements are composed of modal or amodal stimulus attributes.

In the present study, ethanol was administered prior to both training and testing. Therefore, the results obtained may have been due to ethanol's affects on learning and/or performance processes. Ethanol has been shown to affect acquisition (Sahgal et al., 1980) and retrieval (Miller, Adesso, Fleming, Gino, \& Lauerman, 1978) of information. More recently, McKinzie et al. (1994) used similar experimental conditions in a state-dependent design to assess the effects of ethanol on context and CS learning in preweanling rats. Their results determined that, depending on dose and test stimuli, ethanol can affect both acquisition and retrieval processes. For example, the same dose used in the current study had a detrimental influence on context conditioning whether ethanol was given prior to either training or testing, indicating disruption of both acquistion and retrieval processes.

In conclusion, these results provide further evidence that one of the many effects of ethanol in adults is to preferentially reduce the processing of contextual stimuli. This study also suggests that qualitative differences in stimulus selection exist during development. It is proposed that adults usually process stimuli on a modal basis, whereas infants are more likely to encode stimuli in a nonspecific manner and attend to invariant stimulus attributes. In turn, these different modes of encoding are differentially sensitive to the effects of ethanol.

\section{REFERENCES}

Alvarado, M. C., \& Rudy, J. W. (1992). Some properties of configural learning: An investigation of the transverse-patterning problem. Journal of Experimental Psychology: Animal Behavior Processes, 18, 145-153.

Carew, M. B., \& Rudy, J. W. (1991). Multiple functions of context during conditioning: A developmental analysis. Developmental Psychobiology, 24, 191-209.

DANIEL, L. C. (1992). Ethanol effects on central $N$-methyl-D-aspartate receptors. In R. R. Watson (Ed.), Alcohol and neurobiology: Receptors, membranes, and channels (pp. 13-22). Boca Raton, FL: CRC Press.

DEVENPORT, L. D. (1984). Extinction-induced spatial dispersion in the radial arm maze: Arrest by ethanol. Behavioral Neuroscience, $\mathbf{9 8}$, 979-985.

DevenPort, L. D., \& Cater, N. (1986). Ethanol blockade of context change effects. Behavioral \& Neural Biology, 45, 135-142.

Devenport, L. D., \& Hale, R. L. (1989). Contributions of hippocampus and neocortex to the expression of ethanol effects. Psychopharmacology, 99, 337-344.

Devenport, L. D., \& Merriman, B. J. (1983). Ethanol and behavioral variability in the radial-arm maze. Psychopharmacology, 79, 21-24.

Devenport, L. D., Merriman, B. J., \& Devenport, J. A. (1983). Effects of ethanol on enforced spatial variability in the 8-arm radial maze. Pharmacology, Biochemistry \& Behavior, 18, 55-59.

Devenport, L. D., Stidham, J., \& Hale, R. (1989). Ethanol and spatial localization. Behavioral Neuroscience, 103, 1259-1266.

Erblich, J., \& Earleywine, M. (1995). Distraction does not impair memory during intoxication: Support for the attention-allocation model. Journal of Studies on Alcohol, 56, 444-448.

GIBSON, W. E. (1985). Effects of alcohol on radial maze performance in rats. Physiology \& Behavior, 35, 1003-1005.

HAYs, W. L. (1988). Statistics. Orlando, FL: Holt, Rinehart \& Winston. Hinderliter, C. F., \& Misanin, J. R. (1988). Weanling and senescent rats process simultaneously presented odor and taste differently than young adults. Behavioral \& Neural Biology, 49, 112-117.

Hollstedt, C., Olsson, O., \& Rydberg, U. (1980). Effects of ethanol on the developing rat: Il. Coordination as measured by the tilting plane test. Medical Biology, 58, 164-168.

HollstedT, C., \& RydBeRG, U. (1985). Postnatal effects of alcohol on the developing rat. In U. Rydberg (Ed.), Alcohol and the developing rat (pp. 69-84). New York: Raven Press.

Jalava, K. M., Mattila, M. J., Tarssanen, M., \& Vanakoski, J. (1995). Lorazepam and diazepam differently impair divided attention. Pharmacology, Biochemistry \& Behavior, 51, 189-197.

Karmel, B. Z., Gardner, J. M., \& Magano, C. L. (1991). Attention and arousal in early infancy. In M. J. S. Weiss \& P. R. Zelazo (Eds.), Newborn attention: Biological constraints and the influence of experience (pp. 339-376). Norwood, NJ: Ablex.

Kelly, S. J., Bonthius, D. J., \& West, J. R. (1987). Developmental changes in alcohol pharmacokinetics in rats. Alcoholism: Clinical \& Experimental Research, 11, 281-286.

KEPPEL, G. (1982). Design and analysis: A researcher's handbook (2nd ed.). Englewood Cliffs, NJ: Prentice-Hall.

KIM, J. J., \& FANSELOW, M. S. (1992). Modality-specific retrograde amnesia of fear. Science, 256, 675-677.

KuCharsKi, D., \& SPEAR, N. E. (1985). Potentiation of a conditioned taste aversion in preweanling and adult rats. Behavioral \& Neural Biology, 40, 44-57.

LEWKowICZ, D. J. (1994). Development of intersensory perception in human infants. In D. J. Lewkowicz \& R. Lickliter (Eds.), The development of intersensory perception (pp. 165-203). Hillsdale, NJ: Erlbaum.

Lovinger, D., White, G., \& Weight, F. (1989, March 31). Ethanol inhibits NMDA-activated ion current in hippocampal neurons. Science, 243, 1721-1724. 
Lovinger, D., White, G., \& WeIGHT, F. (1990). NMDA receptormediated synaptic excitation selectively inhibited by ethanol in hippocampal slice from adult rat. Journal of Neuroscience, 10, 13721379.

MAIER, D. M., \& Pohorecky, L. A. (1986). The effect of ethanol and sex on radial arm maze performance in rats. Pharmacology. Biochemistry \& Behavior, 25, 703-709.

MARLIN, N. A. (1981). Contextual associations in trace conditioning. Animal Learning \& Behavior, 9, 519-523.

Marriott, J., Alpern, H., \& Crabbe, J. (1974). Alcohol effects on delayed responding by rats. Quarterly Journal of Studies in Alcohol, 35, 45-58.

Mathews, D. B., Simson, P. E., \& Best, P. J. (1996). Acute ethanol impairs spatial memory but not stimulus/response memory in the rat. Alcoholism: Clinical \& Experimental Research, 19, 902-909.

McKinzie, D. L., Lee, J., Bronfen, J. H., Spear, L. P., \& Spear, N. E. (1994). Context and tone conditioning are selectively impaired by ethanol in the preweanling rat: Effects of dose and time of administration. Behavioral \& Neural Biology, 62, 201-209.

McKinziE, D. L., \& SPEAR, N. E. (1995). Ontogenetic differences in conditioning to context and $\mathrm{CS}$ as a function of context saliency and CS-US interval. Animal Learning \& Behavior, 23, 304-313.

Mellon, R. C., Kraemer, P. J., \& SpeaR, N. E. (1991). Intersensory development and Pavlovian conditioning: Stimulus selection and encoding of lights and tones in the preweanling rat. Journal of Experimental Psychology: Animal Behavior Processes, 17, 448-464.

Miller, M. E., Adesso, V. J., Fleming, J. P., Gino, A., \& Lauerman, R. (1978). Effects of alcohol on the storage and retrieval processes of heavy social drinkers. Journal of Experimental Psychology: Human Learning \& Memory, 4, 246-255.

Moye, T. B., \& Rudy, J. W. (1985). Ontogenesis of learning: VI. Learned and unlearned responses to visual stimulation in the infant hooded rat. Developmental Psychobiology, 18, 395-409.

MOYE, T. B., \& RUDY, J. W. (1987). Ontogenesis of trace conditioning in young rats: Dissociation of associative and memory processes. Developmental Psychobiology, 20, 405-414.

MYERS, J. L., \& Well, A. D. (1991). Research design and statistical analysis. New York: HarperCollins.

Phillips, R. G., \& LeDoux, J. E. (1992). Differential contribution of amygdala and hippocampus to cued and contextual fear conditioning. Behavioral Neuroscience, 106, 274-285.

Rajachandran, L., Spear, N. E., \& Spear, L. P. (1993). Effects of the combined administration of the $5 \mathrm{HT}_{3}$ antagonist MDL 72222 and ethanol on conditioning in the periadolescent and adult rat. Pharmacology, Biochemistry \& Behavior, 46, 535-542.

Rupy, J. W. (1991). Elemental and configural associations, the hippocampus and development. Developmental Psychobiology, 24, 221-236.

RUDY, J. W. (1992). Development of learning: From elemental to configural associative networks. Advances in Infancy Research, 7, 247 289.

Rudy, J. W., KeITH, J. R., \& GeOrGen, K. (1993). The effect of age on children's learning of problems that require a configural association solution. Developmental Psychobiology, 26, 171-184.

RUDY, J. W., \& MORLEDGE, P. (1994). Ontogeny of contextual fear conditioning in rats: Implications for consolidation, infantile amnesia, and hippocampal system function. Behavioral Neuroscience, 108, 227-234.

RUdY, J. W., \& SUTherLAND, R. J. (1989). The hippocampal formation is necessary for rats to learn and remember configural discriminations. Behavioural Brain Research, 34, 97-109.

Sahgal, A., Eckberg, S. C., Howell, S. J., \& Iverson, S. D. (1980). Ethanol affects the acquisition of information. Psychopharmacology, 71, 195-200.

SPEAR, N. E., \& KUCHARSKI, D. (1984a). Ontogenetic differences in the processing of multi-element stimuli. In H. Roitblat, T. Bever, \& H. Terrace (Eds.), Animal cognition (pp. 545-567). Hillsdale, NJ: Erlbaum.

SPEAR, N. E., \& KuChaRSKI, D. (1984b). Ontogenetic differences in stimulus selection during conditioning. In R. Kail \& N. E. Spear (Eds.), Comparative perspectives on the development of memory (pp. 227 252). Hillsdale, NJ: Erlbaum.

SPEAR, N. E., \& MCKINZIE, D. L. (1994). Intersensory integration in the infant rat. In D. J. Lewkowicz \& R. Lickliter (Eds.), The development of intersensory perception: Comparative perspectives (pp. 133161). Hillsdale, NJ: Erlbaum.

SPEAR, N. E., McKinZIE, D. L., \& ARNOLd, H. M. (1994). Suggestions from the infant rat about brain dysfunction and memory. In J. Delacour (Ed.), The memory system of the brain (pp. 278-315). Singapore: World Scientific Publishing.

SPEAR, N. E., \& MOLINA, J. C. (1987). The role of sensory modality in the ontogeny of stimulus selection. In N. Krasnegor, E. M. Blass, M. A. Hofer, \& W. P. Smotherman (Eds.), Perinatal development: A psychobiological perspective (pp. 85-110). San Diego: Academic Press.

SWARTZWELDER, H. S., Wilson, W. A., \& TAYyeb, M. I. (1995). Agedependent inhibition of long-term potentiation by ethanol in immature versus mature hippocampus. Alcoholism: Clinical \& Experimental Research, 19, 1480-1485.

TURKEWITZ, G. (1994). Sources of order for intersensory functioning. In D. J. Lewkowicz \& R. Lickliter (Eds.), The development of intersensory perception: Comparative perspectives (pp. 3-17). Hillsdale, NJ: Erlbaum.

Turkewitz, G., Lewkowicz, D. J., \& Gardner, J. M. (1983). Determinants of infants' perception. In J. S. Rosenblatt, R. A. Hinde, C. Beer, \& M. C. Bushnel (Eds.), Advances in the study of behavior (Vol. 13, pp. 39-62). New York: Academic Press.

TuRKewitz, G., \& Mellon, R. C. (1989). Dynamic organization of intersensory function. Canadian Journal of Psychology, 43, 286301 .

(Manuscript received April 16, 1996; revision accepted for publication July $24,1996$. ) 